The equivariant inverse problem and the Maxwell equations

R. J. Noriega, and C. G. Schifini

Citation: Journal of Mathematical Physics 28, 815 (1987); doi: 10.1063/1.527568

View online: https://doi.org/10.1063/1.527568

View Table of Contents: http://aip.scitation.org/toc/jmp/28/4

Published by the American Institute of Physics

PHYSICS TODAY

WHITEPAPERS
MANAGER'S GUIDE

Accelerate R\&D with Multiphysics Simulation
READ NOW

PRESENTED BY VD $\subset$ MSOL 


\title{
The equivariant inverse problem and the Maxwell equations
}

\author{
R. J. Noriega and C. G. Schifini \\ Departamento de Matemática, Facultad de Ciencias Exactas y Naturales, Universidad de Buenos Aires, \\ 1428 Buenos Aires, Argentina
}

(Received 24 June 1986; accepted for publication 26 November 1986)

In affirmative, the equivariant inverse problem for Maxwell-type Euler-Lagrange expressions is solved. This allows the proof of the uniqueness of the Maxwell equations.

\section{INTRODUCTION}

It is very well known that Maxwell equations can be written in covariant form as ${ }^{1}$

$$
\begin{aligned}
& F^{i j}{ }_{1 j}=J^{i}, \\
& { }^{*} F^{i j}{ }_{\mid j}=0,
\end{aligned}
$$

where $F^{i j}$ is a skew-symmetric tensor and ${ }^{*} F^{i j}=\eta^{i j k} F_{h k}$. Equation (1.2) is equivalent to the existence of a covector field $\psi_{i}$ such that

$$
F_{i j}=\psi_{i, j}-\psi_{j, i} \text {. }
$$

The Maxwell equations can be deduced from a variational principle as follows. If

$$
L=L\left(g_{i j} ; \psi_{i} ; \psi_{i, j}\right) \text {, }
$$

then from a variation of $\psi_{i}$ we obtain as Euler-Lagrange equations,

$$
E^{i}(L)=\frac{\partial L}{\partial \psi_{i}}-\frac{\partial}{\partial x^{j}}\left(\frac{\partial L}{\partial \psi_{i, j}}\right)=0 ;
$$

on choosing

$$
L=\sqrt{g} F^{i j} F_{i j},
$$

Eqs. (1.5) become (1.1).

The left-hand side of (1.1) has two properties of covariance: (1) by a transformation of coordinates it changes as a vector; and (2) by a change of gauge, i.e., by a transformation of the type $\psi_{i} \rightarrow \psi_{i}+\phi_{, i}$, where $\phi$ is a scalar, it is invariant. These properties are also possessed by the Lagrangian (1.6). However, the last assertion is not mandatory since the Lagrangian does not have, in general, any physical meaning, although the Euler-Lagrange expressions do have a meaning. The main purpose of this article is to prove that the situation already encountered with the Maxwell equations is found always in spaces of dimension 4, i.e., the assumption of the two covariance properties for the Euler-Lagrange expressions implies that the Lagrange is equivalent to (it has the same Euler-Lagrange expressions as) a Lagrangian with the same properties. ${ }^{2}$ This solves for the affirmative the equivariant inverse problem ${ }^{3}$ for Maxwell-type Euler-Lagrange expressions.

Precisely, we consider a quantity

$$
B^{i}=B^{i}\left(g_{i j} ; g_{i j, h} ; \psi_{i} ; \psi_{i, j} ; \psi_{i, j h}\right)
$$

such that

(i) $B^{i}$ is a vector,

(ii) $B^{i}$ is gauge invariant,
Here we do not assume any covariance property for $L_{1}$ with respect to transformations of coordinates or changes of gauge. We will prove that (1.7) and (1.8) imply the existence of $L=L\left(g_{i j} ; \psi_{i} ; \psi_{i, j}\right)$, which is a gauge-invariant scalar density such that $E^{i}(L)=B^{i}$.

\section{THE EQUIVARIANT INVERSE PROBLEM}

The condition (iii) in (1.8) written in full is

$$
\begin{aligned}
B^{i}= & L^{i}-L^{i, j ; h k} g_{h k, j}-L^{i, j, h} \psi_{h, j} \\
& -\frac{1}{2}\left(L^{i, j, h, k}+L^{i, k ; h, j}\right) \psi_{h, k j},
\end{aligned}
$$

where $L^{i}=\partial L / \partial \psi_{i}, L^{i, j}=\partial L / \partial \psi_{i, j}$, and $L^{h k}=\partial L / \partial g_{h k}$. The coefficients of (2.1) depend only on $g_{i j}, \psi_{i}$, and $\psi_{i, j}$.

Since $B^{i}$ is gauge invariant, by the replacement theorem ${ }^{4}$ we have

$$
B^{i}=H^{i}-\frac{1}{2} H^{i j h} F_{h j}-\frac{2}{3} H^{i j h k} F_{h k \mid j},
$$

where

$$
\begin{aligned}
& H^{i}=L^{i}\left(g_{i j} ; 0 ; \frac{1}{2} F_{i j}\right), \quad H^{i j h}=L^{i, j ; h}\left(g_{i j} ; 0 ; \frac{1}{2} F_{i j}\right), \\
& H^{i j h k}=\frac{1}{2}\left(L^{i, j ; h, k}+L^{i, k ; h, j}\right)\left(g_{i j} ; 0 ; \frac{1}{2} F_{i j}\right) .
\end{aligned}
$$

Since $B^{i}$ is a tensorial density, the same is true for $\partial B^{i} / \partial \psi_{h, k j}$. From (2.1) this derivative is $\frac{1}{2}\left(L^{i, j ; h, k}+L^{i, k ; h, j}\right)$. Then $H^{i j h k}$ defined by $(2.3)$ is also a tensorial density. Hence, by (2.2), $H^{i}-\frac{1}{2} H^{i j h} F_{h j}$ is a vector density and depends only on $g_{i j}$ and $F_{i j}$. It is known ${ }^{5}$ that such vector densities are zero, and so we obtain

$$
B^{i}=-\frac{2}{3} H^{i j h k} F_{h k \mid j} \text {. }
$$

Denoting $W^{i j h k}=\frac{1}{2}\left(L^{i, j ; h, k}+L^{i, k ; h, j}\right)$, from (2.1) and (2.2) we deduce

$$
W^{i j h k} \psi_{h, k j}=H^{i j h k}\left(\psi_{h, k j}-\psi_{k, h j}\right)+(\cdots),
$$

where in $(\cdots)$ we have gathered the terms that do not depend on $\psi_{h, k j}$. From the equality of the cross derivatives it is easily obtained that $W^{i j h k}=W^{h k i j}$, and by (2.3), $H^{i j h k}$ $=H^{h k i j}$. Also, $W^{i j h k}=W^{i k h j}$ is valid and so $H^{i j h k}=H^{i k h j}$.

Differentiating (2.5) with respect to $\psi_{a, b c}$ and taking into account the mentioned symmetries, we have

$$
2 W^{i c a b}=2 H^{i c a b}-H^{i c b a}-H^{i b c a} .
$$

Changing $i$ with $a$ and $c$ with $b$ in (2.6) and subtracting the resulting equation to (2.6), it follows that

$$
H^{a b c i}+H^{a c b i}-H^{i c b a}-H^{i b c a}=0 .
$$

Changing $a$ with $c$ and $b$ with $i$ in (2.7) and subtracting the resulting equation to (2.7), we have $H^{a c b i}=H^{i b c a}$, or else

$$
H^{a c b i}=H^{c a i b} \text {. }
$$




\section{Denoting}

$$
T^{i j h k}=-\frac{1}{3}\left(H^{i j h k}-H^{i j k h}\right),
$$

it follows that $T^{i j h k}$ is a tensor, such that

$$
B^{i}=\sqrt{g} T^{i j h k} F_{h k \mid j},
$$

and besides,

$$
T^{i j h k}=-T^{j i h k}=T^{h k i j} .
$$

Using Theorem 3 in Kerrighan, ${ }^{5}$ we deduce

$$
\begin{aligned}
T^{i j h k}= & A * F^{i j *} F^{h k}-B\left(F^{i k}{ }^{*} F^{j h}-F^{i h} * F^{j k}\right. \\
& \left.-F^{j k}{ }^{*} F^{i h}+F^{j h} * F^{i k}\right)+C F^{i j} F^{h k} \\
& +2 D\left(g^{i h} g^{j k}-g^{i k} g^{j h}\right)+E \eta^{i j h k}
\end{aligned}
$$

where $A, B, C, D$, and $E$ are scalar concomitants of $g_{i j}$ and $F_{i j}$. Then

$$
\begin{aligned}
B^{i}= & \sqrt{g}\left\{\left(A^{*} F^{i j *} F^{h k}+B\left(F^{i j *} F^{h k}+{ }^{*} F^{i j} F^{h k}\right)\right.\right. \\
& \left.\left.+C F^{i j} F^{h k}\right) F_{h k \mid j}+D F_{\mid j}^{i j}\right\} .
\end{aligned}
$$

Since $B^{i}$ is a Euler-Lagrange expression, it satisfies certain identities ${ }^{6}$ :

$$
\begin{aligned}
& B^{i ; j, h k}=B^{j ; i, h k}, \\
& B^{i ; j, h}=-B^{j ; i, h}+2 \frac{\partial}{\partial x^{k}}\left(B^{j ; i, h k}\right), \\
& B^{i ; j}=B^{j ; i}-\frac{\partial}{\partial x^{h}}\left(B^{j ; i, h}\right)+\frac{\partial^{2}}{\partial x^{h} \partial x^{k}}\left(B^{j ; i, h k}\right) .
\end{aligned}
$$

It is easy to see that (2.14) is satisfied identically. Let us see what restrictions (2.15) imposes on $A, B, C$, and $D$. It is known ${ }^{7}$ that there are functions $\lambda_{1}, \lambda_{2}, \lambda_{3}$, and $\lambda_{4}$ of two real variables such that

$$
\begin{array}{ll}
A=\lambda_{1}(\phi, \psi), & B=\lambda_{2}(\phi, \psi), \\
C=\lambda_{3}(\phi, \psi), & D=\lambda_{4}(\phi, \psi) ;
\end{array}
$$

where

$$
\phi={ }^{*} F^{i j} F_{i j}, \quad \psi=F^{i j} F_{i j} .
$$
lowing:

Equation (2.15) gives rise, in view of (2.13), to the fol-

$$
\begin{aligned}
& \left\{2 \frac{\partial \lambda_{1}}{\partial \psi}\left(F^{h k} * F^{a c} * F^{i b}-F^{a b} * F^{i c} * F^{h k}\right)+2 \frac{\partial \lambda_{2}}{\partial \psi}\left(F^{h k} F^{a c} * F^{i b}-F^{a b} F^{i c} * F^{h k}\right)+2 \frac{\partial \lambda_{2}}{\partial \phi}\left({ }^{*} F^{h k} F^{a b *} F^{i c}-{ }^{*} F_{a b} * F^{i c} F^{h k}\right)\right. \\
& \quad+2 \frac{\partial \lambda_{2}}{\partial \phi}\left({ }^{*} F^{h k} F^{a c} F^{i b}-{ }^{*} F^{a b} F^{i c} F^{h k}\right)+\frac{\partial \lambda_{4}}{\partial \phi}\left({ }^{*} F^{h k} g^{a i} g^{c b}-{ }^{*} F^{h k} g^{a b} g^{c i}-2 * F^{a b} g^{h i} g^{k c}\right) \\
& \quad+\frac{\partial \lambda_{4}}{\partial \psi}\left(F^{h k} g^{a i} g^{c b}-F^{h k} g^{a b} g^{c i}-2 F^{a b} g^{h i} g^{k c}\right)+\lambda_{2}\left({ }^{*} F^{i b} g^{h a} g^{k c}+\frac{1}{2} F^{h k} g^{i b} g^{c a}-\frac{1}{2} F^{h k} g^{i a} g^{c b}\right) \\
& \left.+\lambda_{3}\left(F^{i b} g^{h a} g^{k c}+\frac{1}{2} F^{h k} g^{i b} g^{c a}-\frac{1}{2} F^{h k} g^{i a} g^{c b}\right)\right\}\left(\psi_{h, k c}-\psi_{k, h c}\right)+(i \leftrightarrow a)=(\cdots),
\end{aligned}
$$

where $(i \leftrightarrow a)$ denotes symmetrization of the previous expression with respect to $i$ and $a$, and where we have gathered in $(\cdots)$ all the terms that do not depend on the second derivatives of $\psi_{i}$.

It is known ${ }^{8}$ that given a point on the underlying manifold there is a coordinate system such that, at the point

$$
\begin{aligned}
\left(g_{i j}\right) & =\left(\begin{array}{cccc}
-1 & 0 & 0 & 0 \\
0 & 1 & 0 & 0 \\
0 & 0 & 1 & 0 \\
0 & 0 & 0 & 1
\end{array}\right), \\
\left(F_{i j}\right) & =\left(\begin{array}{cccc}
0 & \alpha & 0 & 0 \\
-\alpha & 0 & 0 & 0 \\
0 & 0 & 0 & \beta \\
0 & 0 & -\beta & 0
\end{array}\right),
\end{aligned}
$$

from where we deduce

$$
\begin{aligned}
& \left(F^{i j}\right)=\left(\begin{array}{cccc}
0 & -\alpha & 0 & 0 \\
\alpha & 0 & 0 & 0 \\
0 & 0 & 0 & \beta \\
0 & 0 & -\beta & 0
\end{array}\right), \\
& \left({ }^{*} F^{i j}\right)=\left(\begin{array}{cccc}
0 & 2 \beta & 0 & 0 \\
-2 \beta & 0 & 0 & 0 \\
0 & 0 & 0 & 2 \alpha \\
0 & 0 & -2 \alpha & 0
\end{array}\right) .
\end{aligned}
$$

Differentiating (2.19) with respect to $\psi_{a, b c}$ and choosing $i=a=1, c=b=s=4$, and $r=3$, we obtain

$$
4 \alpha \frac{\partial \lambda_{4}}{\partial \phi}+2 \beta \frac{\partial \lambda_{4}}{\partial \psi}=2 \alpha \lambda_{2}+\beta \lambda_{3} \text {. }
$$

Differentiating (2.19) with respect to $\psi_{a, b c}$ and choosing $i=a=4, c=b=s=2$, and $r=1$, we have

$$
4 \beta \frac{\partial \lambda_{4}}{\partial \phi}-2 \alpha \frac{\partial \lambda_{4}}{\partial \psi}=2 \beta \lambda_{2}-\alpha \lambda_{3} .
$$

Let us suppose for the moment that $\operatorname{det}\left(F_{i j}\right) \neq 0$. Then from (2.22) and (2.23) it follows easily that

$$
\lambda_{2}=2 \frac{\partial \lambda_{4}}{\partial \phi}, \quad \lambda_{3}=2 \frac{\partial \lambda_{4}}{\partial \psi} .
$$

Differentiating (2.19) with respect to $\psi_{a, b c}$ and choosing now $a=b=1, c=i=2, r=3$, and $s=4$, we obtain

$$
2 \beta \frac{\partial \lambda_{2}}{\partial \phi}+\alpha \frac{\partial \lambda_{2}}{\partial \psi}=2 \beta \frac{\partial \lambda_{1}}{\partial \psi}+\alpha \frac{\partial \lambda_{3}}{\partial \phi} .
$$

Finally, differentiating (2.19) with respect to $\psi_{a, b c}$ and choosing $a=b=4, c=i=3, r=1$, and $s=2$, we have

$$
2 \alpha \frac{\partial \lambda_{1}}{\partial \psi}+\beta \frac{\partial \lambda_{2}}{\partial \psi}=2 \alpha \frac{\partial \lambda_{2}}{\partial \phi}+\beta \frac{\partial \lambda_{3}}{\partial \phi} .
$$

From (2.25) and (2.26) it follows that

$$
\frac{\partial \lambda_{2}}{\partial \phi}=\frac{\partial \lambda_{1}}{\partial \psi}, \quad \frac{\partial \lambda_{2}}{\partial \psi}=\frac{\partial \lambda_{3}}{\partial \phi} .
$$

Now, (2.24) and (2.27) are the integrability conditions we 
need to establish the existence of a scalar $T=T(\phi, \psi)$ such that

$$
\begin{array}{ll}
\lambda_{1}=2 \frac{\partial^{2} T}{\partial \phi^{2}}, & \lambda_{2}=2 \frac{\partial^{2} T}{\partial \phi \partial \psi}, \\
\lambda_{3}=2 \frac{\partial^{2} T}{\partial \psi^{2}}, & \lambda_{4}=\frac{\partial T}{\partial \psi} .
\end{array}
$$

In this case, a straightforward computation proves that

$$
B^{i}=E^{i}(L) \text {, }
$$

where $L=\sqrt{g} T$ is a gauge-invariant scalar density. This solves the equivariant inverse problem since $\operatorname{det}\left(F_{i j}\right) \neq 0$ is a dense subset of the space of variables $g_{i j}, F_{i j}$ [and then (2.29) is valid everywhere by a continuity argument ].

\section{THE MAXWELL EQUATIONS}

We have proved in Sec. II that if $B^{i}$ is of the type (1.7) and it satisfies (1.8), then

$$
B^{i}=L^{i j}{ }_{\mid j},
$$

where $L^{i j}=\partial L / \partial F_{i j}$ and $L=\sqrt{g} T(\phi, \psi)$, with $\phi$ and $\psi$ given by (2.18). By similarity, with the Maxwell equations (1.1) and (1.2), we could claim the field equations to be

$$
\begin{aligned}
& L^{i j}{ }_{\mid j}=J^{i}, \\
& { }^{*} L^{i j}{ }_{1 j}=0 .
\end{aligned}
$$

Now, Eqs. (3.3) corresponding to the Maxwell internal equations do not depend on the charge and current distribution, and so they should be satisfied identically. Written out in full, they are

$$
\begin{aligned}
* L^{i j}{ }_{1 j}= & \sqrt{g}\left[16 \frac{\partial^{2} L}{\partial \phi^{2}}{ }^{*} F^{h k} F^{i j} F_{h k \mid j}+16 \frac{\partial^{2} L}{\partial \phi \partial \psi} F^{h k} F^{i j} F_{h k \mid j}\right. \\
& +8 \frac{\partial L}{\partial \phi} F^{i j}{ }_{1 j}+4 \frac{\partial^{2} L}{\partial \psi \partial \phi} * F^{h k} * F^{i j} F_{h k \mid j} \\
& \left.+4 \frac{\partial^{2} L}{\partial \psi^{2}} * F^{i j} F^{h k} F_{h k \mid j}\right]=0 .
\end{aligned}
$$

Differentiating (3.4) with respect to $\psi_{a, b c}$, we obtain

$$
\begin{aligned}
4 \frac{\partial^{2} L}{\partial \phi^{2}}\left({ }^{*} F^{a b} F^{i c}+{ }^{*} F^{a c} F^{i b}\right)+4 \frac{\partial^{2} L}{\partial \phi \partial \psi}\left(F^{a b} F^{i c}+F^{a c} F^{i b}\right) \\
+\frac{\partial^{2} L}{\partial \psi \partial \phi}\left({ }^{*} F^{a b} F^{i c}+{ }^{*} F^{a c}{ }^{*} F^{i b}\right) \\
\quad+\frac{\partial^{2} L}{\partial \psi^{2}}\left(F^{a b *} F^{i c}+F^{a c} F^{i b}\right) \\
+\frac{\partial L}{\partial \phi}\left(2 g^{i a} g^{b c}-g^{i b} g^{a c}-g^{i c} g^{a b}\right)=0 .
\end{aligned}
$$

In the coordinate system in which $(2.20)$ is valid and choosing $a=b=1, c=i=3$, we have $\partial L / \partial \phi=0$ if we assume $\operatorname{det}\left(F_{i j}\right) \neq 0$. Choosing now $a=i=1, b=c=2$, it follows that $\partial^{2} L / \partial \psi^{2}=0$. By a continuity argument we deduce that everywhere,

$$
L=\sqrt{g}(C \psi+A)
$$

where $A$ and $C$ are real numbers, and so

$$
L{ }^{i j}{ }_{\mid j}=C \sqrt{g} F^{i j}{ }_{1 j},
$$

which makes (3.2) the usual Maxwell equations.

\section{ACKNOWLEDGMENT}

We thank the referee for helpful suggestions.

'The tensor $g_{i j}$ is the metric tensor of a four-space-time manifold and $g=\left|\operatorname{det}\left(g_{i j}\right)\right|$. We use the summation convention and we raise and lower indices with $g_{i j}$ and $g^{i j}$. We denote $\eta^{i h k}=(\sqrt{g})^{-1} e^{j h k}$, where $e^{j h k}$ are the Levi-Civita permutation symbols. The vertical bar stands for covariant derivative and the comma stands for the usual partial derivative. Here, $J^{i}$ is the charge-current vector.

${ }^{2}$ This is not true for all dimensions. If it is 3 , then $B^{i}=\epsilon^{i n h} F_{i n}$ is of the form (1.7) and satisfies (1.8) with $L=\frac{1}{2} e^{i h} \psi_{i} F_{j h}$, but $L$ cannot be replaced by a gauge invariant Lagrangian. See, for example, M. C. Calvo, C. Lopez, R. J. Noriega, and C. G. Schifini, "Gauge invariance of Euler-Lagrange expressions in Einstein-Yang-Mills field theories," submitted to Gen. Relativ. Gravit.

${ }^{3}$ I. M. Anderson, Ann. Math. 120, 329 (1984).

${ }^{4}$ G. W. Horndeski, Tensor (N.S.) 32 (2), 131 (1978).

${ }^{5}$ B. Kerrighan, Gen. Relativ. Gravit. 13 (1), 19 (1981).

${ }^{6} I$. M. Anderson and T. Duchamp, Am. J. Math. 102 (5), 781 (1980).

${ }^{7}$ R. J. Noriega and C. G. Schifini, Gen. Relativ. Gravit. 16 (3), 292 (1984).

${ }^{8}$ V. Hlavatý, J. Rat. Mech. Anal. 1, 539 (1952). 